\title{
In Situ and High Resolution TEM Studies of Nano-scale Materials
}

\author{
R. Sinclair *, A.L. Koh * \#, P.J. Kempen * and H.J. Jung * \\ * Department of Materials Science and Engineering, Stanford University, Stanford, CA 94305, \\ United States \\ \# Department of Materials, Imperial College London, London SW72AZ, United Kingdom
}

Our first review of the application of in situ, high-resolution transmission electron microscopy (TEM) to study the behavior of materials at controlled, elevated temperatures appeared in 1988 [1]. That paper chronicled the development of this technique from using electron beam heating (as others had also done at that time) to actually employing a stable specimen heating holder without degrading the quality of the high resolution images. Of particular importance was the demonstration that kinetically driven events in many systems occurred at the same rate and with the same activation energy as those in bulk materials. This led to a range of such studies on reactions on thin films, such as solid state amorphization at metal-semiconductor interfaces (eg. [2,3]), metal-induced crystallization of amorphous semiconductors (eg. [4]), multilayer reactions ([eg. 5,6]) and most recently amorphous gate oxide crystallization [7]. This method is now being applied for examining processes in nano-materials (eg. [8-10]).

Our current work is focused on the stability and functionalization of magnetic and noble metal nanoparticles for applications in cancer detection and therapy. Thus a method has been described to produce elemental (eg. Fe, Co) and alloy (eg. FeCo, FeAu) superparamagnetic nanoparticles encapsulated by graphite which remain unchanged after several years at room temperature and pressure (eg. [11]). Complementing this, we have investigated the structure of silver surface enhanced Raman (SERS) nanoparticles functionalized with antibodies. Figs. 1 and 2 compare typical negatively stained TEM images (eg. [12]) with energy filtered TEM images taken using the carbon $\mathrm{K}$ edge. The latter clearly shows the carbon-based biological coating much more effectively than does the conventional imaging. This article therefore reviews these works [13].

\section{References}

[1] R. Sinclair et al., Acta Crystallogr. Sec. A 44 (1988), 965-975.

[2] D.H. Ko and R. Sinclair, Ultramicroscopy 54 (1994), 166-178.

[3] H. Tanaka et al, J. Appl. Phys. 78 (1995), 4982-4987.

[4] T.J. Konno and R. Sinclair, Philos. Mag. B 71 (1995), 163-178.

[5] K. Holloway and R. Sinclair, J. Less Common Metals, 140 (1988), 139-148.

[6] H.-J. Lee et al., Acta Mater. 47(15/16) (1999), 3965-3975.

[7] K.-H. Min et al., Philos. Mag., 85(18) (2005), 2049-2063.

[8] R. Sharma et al., Nanotechnology 18 (2007), 125602-125609.

[9] H. Adhikari et al., ACS Nano 1(5) (2007), 415-422.

[10] J.E. Wittig et al., Micros. Microanal. 14 Suppl. 2 (2008), 216CD-217CD.

[11] H. Li et al., Micros. Microanal. 12 Suppl. 2 (2006) 524CD-525CD.

[12] A.L. Koh et al., Ultramicroscopy 109 (2008) 110-121.

[13] Funding for our recent works is kindly provided by the Center for Cancer Nanotechnology Excellence Focused on Therapy Response (CCNE-TR) grant (NIH U54. Use of the facilities at the Stanford Nanocharacterization Laboratory and the National Center for Electron 
Microscopy, Lawrence Berkeley Lab, which is supported by the U.S. Department of Energy under Contract \# DE-AC02-05CH11231, is recognized.

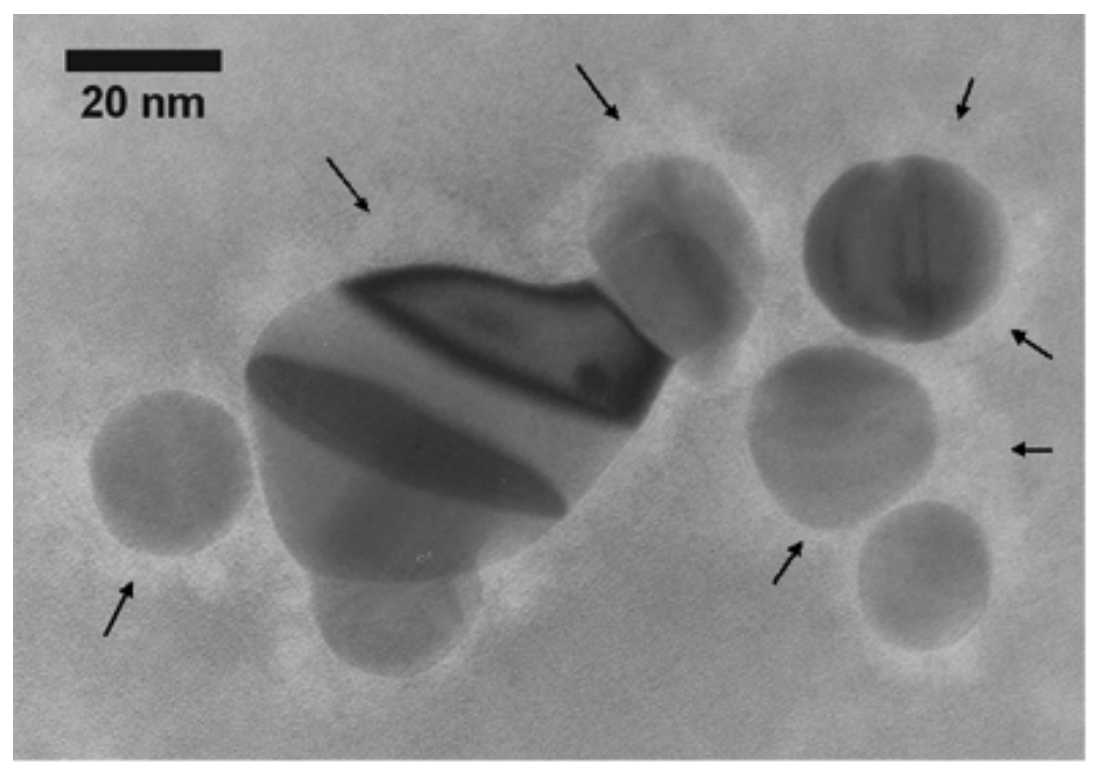

Fig. 1. Bright field TEM image of negatively stained silver nanoparticles coated with bovine serum albumin (BSA) and glutaraldehyde, and functionalized with CD54 antibodies. The light halos around each silver nanoparticle arise for the biological matter.

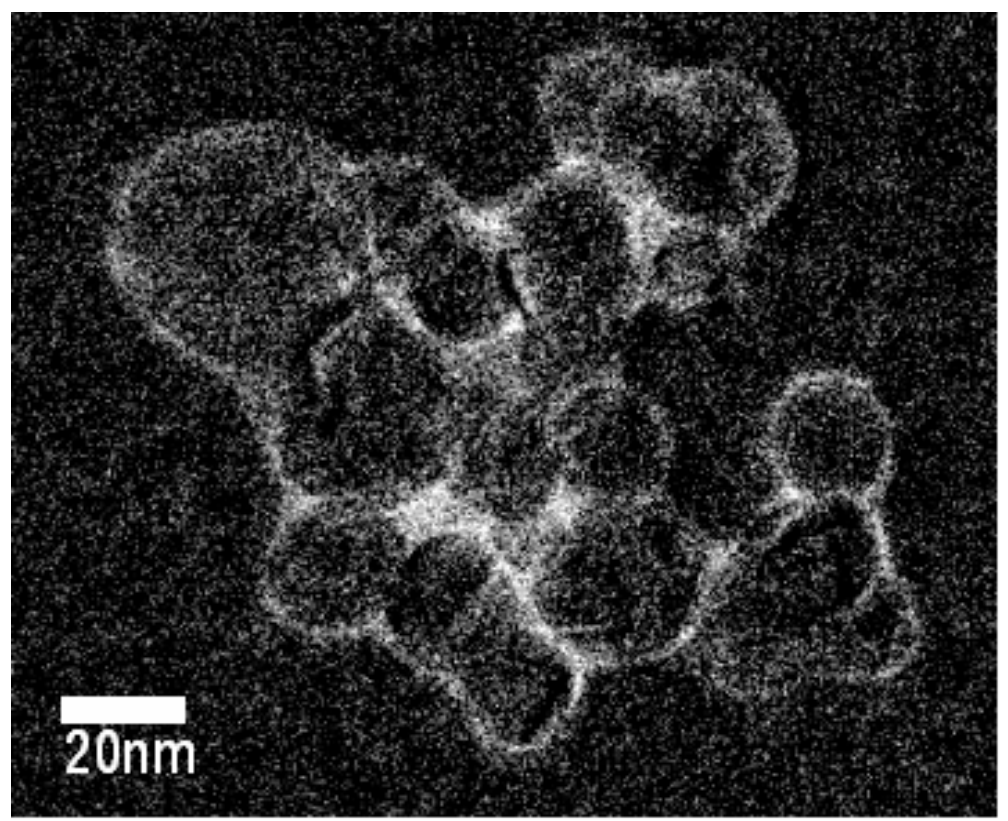

Fig. 2. Energy filtered TEM image using the carbon $\mathrm{K}$ edge of $284 \mathrm{eV}$ showing the biological coating of SERS silver nanoparticles. 